\title{
Asymmetric Fabry-Perot-type transmission in photonic-crystal gratings with one-sided corrugations at a two-way coupling
}

\author{
Andriy E. Serebryannikov, ${ }^{1, *}$ K. B. Alici, ${ }^{2}$ Thore Magath, ${ }^{3}$ A. Ozgur Cakmak,${ }^{4}$ and Ekmel Ozbay ${ }^{4}$ \\ ${ }^{1}$ Technische Universität Hamburg-Harburg, E-3, D-21071 Hamburg, Germany \\ ${ }^{2}$ Department of Physics and Center for Nano and Molecular Science and Technology, The University of Texas at Austin, \\ Austin, Texas 78712, USA \\ ${ }^{3}$ European Technology Center, Panasonic Electronic Devices Europe GmbH, D-21337 Lueneburg, Germany \\ ${ }^{4}$ Nanotechnology Research Center, Bilkent University, 06800 Ankara, Turkey
}

(Received 24 January 2012; revised manuscript received 24 September 2012; published 29 November 2012)

\begin{abstract}
Strongly asymmetric Fabry-Perot-type transmission arising at the two-way coupling has been studied in the case of normal incidence for slabs of two-dimensional photonic crystals (PCs) with one-sided corrugations that are made of linear isotropic materials. Comparing to the scenario of unidirectional transmission known for the structures with broken spatial inversion symmetry that requires zero order being uncoupled, in the studied mechanism zero order is either the sole order or one of the orders that may be coupled to a Floquet-Bloch mode. Contrary to the earlier studies of asymmetric transmission at the coupled zero order, structures with nondeep corrugations are considered, which allow one to combine Fabry-Perot-type total-transmission maxima with diffractions in a desired way. At a proper choice of PC lattice and corrugation parameters, higher orders can dominate in Fabry-Perot-type transmission at the noncorrugated-side illumination and also at the total-transmission maxima, whereas only zero order contributes to the transmission at the corrugated-side illumination. As a result, strong asymmetry can be obtained without uncoupling of zero order but it invokes the unidirectional contribution of higher orders. The presented results show that the entire structure can be approximately decomposed into the two independent, regular and grating (nonregular), parts whose contributions to the transmission are additive. Multiple asymmetric transmission maxima can coexist with a rather high equivalent group index of refraction. Possible applications of the studied transmission mechanism are discussed.
\end{abstract}

DOI: 10.1103/PhysRevA.86.053835

PACS number(s): 42.25.Fx, 42.25.Bs, 42.79.Dj, 78.20.Ci

\section{INTRODUCTION}

Finite-thickness slabs of photonic crystals (PCs) with corrugated interface(s), which are known also as PC gratings, have recently been suggested [1-5]. They enable transmission [3-5] and reflection [1,2] regimes that significantly differ from those achievable for noncorrugated slabs of PC. An even bigger class of transmission regimes has recently been studied that is associated with a strong forward-to-backward transmission contrast in the structures that contain anisotropic [6,7] or nonlinear [8] materials. Various periodic structures, e.g., gratings supporting surface plasmons, metamaterials, and multilayers $[9,10]$, can be used to obtain these regimes. The response of the utilized artificial structures depends on the geometry, content, and period of the unit cell [11,12]. Interest has been growing in the partial analogs of diodelike but still reciprocal transmission in these structures, which only contain linear and isotropic constituents and, hence, do not need the biasing fields. In PC gratings, these regimes are connected with the common effect of the peculiar dispersion of Floquet-Bloch modes in PC and diffractions at the corrugated interface(s). Among them, wideband unidirectionality with strong higherorder transmission at the corrugated-side illumination and vanishing transmission at the noncorrugated-side illumination is considered to be the most promising operation regime $[4,13]$.

Diffraction-inspired unidirectional transmission has been demonstrated theoretically and experimentally for nonsymmetric structures, which are based on PCs, in a wide

*serebryannikov@tu-harburg.de frequency range that extends from the acoustic to the optical frequencies [14-17]. Compared to the thin structures based on subwavelength resonators [18], which also have broken spatial inversion symmetry and, thus, may show asymmetric transmission, PC gratings need anomalous diffractions rather than polarization conversion. It is noteworthy that the conventional isotropic dielectric material has dispersion that is inconsistent with suppression of higher diffraction orders within a wide frequency range, so that asymmetry in transmission through a nonsymmetric grating made of such a material appears while the zero-order (symmetric) component of the transmission is nonvanishing.

Fabry-Perot resonances are known in PCs in the diffractionfree (zero-order), nonchanneled transmission, and in the channeled waveguide regime (e.g., see Refs. [19-22]). The former can appear due to either regular Floquet-Bloch modes of PCs or defect modes in PCs with structural defects. The latter is associated with propagation along the $\operatorname{defect}(\mathrm{s})$. Furthermore, the mentioned modes can show dispersion that corresponds to a high group index of refraction [22-25].

In this paper, we study asymmetric Fabry-Perot mechanism relevant transmission in nonsymmetric PC gratings, which are made of linear isotropic dielectrics and illuminated by the normally incident $s$-polarized plane waves. Consideration is focused on the structures, which are based on two-dimensional square-lattice dielectric PCs. In most of the studied performances we use the simplest corrugations, which are obtained by removing every second rod from one of the interface layers of the rods of the corresponding noncorrugated slab of PC. It is shown that strong asymmetry in transmission can be obtained via the dominant contribution of higher diffraction 
orders at the noncorrugated-side illumination. Contrary to earlier studies of the PC gratings, where strong asymmetry in transmission is associated with only higher orders being coupled to one of the Floquet-Bloch waves and only at the corrugated-side illumination, we study here the case when zero order, which is associated with the symmetric component of transmission, is coupled to a Floquet-Bloch wave. Total transmission is achieved in the studied Fabry-Perot-type (reflection-free) regime only if the noncorrugated interface of a PC grating is illuminated. Thus, the open zero-order transmission channel in PC is itself a two-way transmission (i.e., it is not asymmetric), while the strong asymmetry in transmission appears due to the difference in the coupling and diffractions at the corrugated and noncorrugated interfaces. This is the principal difference between the studied mechanism and that requiring zero order to be uncoupled [4,13,15,26]. Asymmetry in transmission at the Fabry-Perot resonances is also expected to appear at the uncoupled zero order. However, the coupled zero-order regime, which is the focus of this paper, can be even more interesting at least because of the possible appearance of Fabry-Perot resonances in both forward transmission (corrugated-side illumination) and backward transmission (noncorrugated-side illumination) [27].

We show that the studied mechanism can be obtained at the different types of dispersion of Floquet-Bloch modes and coupling scenarios. In particular, it is not necessary that zero order is the only order allowed to be coupled. Higher orders may formally be coupled at the corrugated input interface, but their contribution to the transmission can be negligible. Connection between the equivalent group index of refraction and the extent to which asymmetry in transmission is pronounced is discussed. At a proper choice of the PC grating parameters, one-way splitting with total transmission in one direction, dual-band diodelike operation with the nearly opposite directions of strong transmission in the neighboring frequency ranges, and switching between total zero-order transmission and strong first-order transmission can be realized. The presented transmission results have been obtained by using the coupled-integral-equation technique [28]. CST Microwave Studio, a commercial solver based on the finite integration method [29], has been used to calculate dispersion of Floquet-Bloch modes.

\section{THEORETICAL BACKGROUND}

Total-transmission maxima connected with Fabry-Perot resonances are well known for slabs that are made of linear, isotropic, homogeneous, lossless materials. In this case, transmittance is given by the Airy formula [30]:

$$
T=\frac{(1-r)^{2}}{(1-r)^{2}+4 r \sin ^{2}\left(N^{\prime} k D \cos \theta^{\prime}\right)},
$$

where $r=\sin ^{2}\left(\theta-\theta^{\prime}\right) / \sin ^{2}\left(\theta+\theta^{\prime}\right)$ is the reflectance of a slab-air interface, $\sin \theta^{\prime}=\sin \theta / N^{\prime}, \theta$ is the angle of incidence, $\theta^{\prime}$ is the angle of refraction, $D$ is the thickness of the slab, $N^{\prime}$ is the index of refraction of the slab material, and $k$ is the free-space wave number. The maxima of $T=1$ correspond to $N^{\prime} k D \cos \theta^{\prime}=m \pi, m=1,2, \ldots$.

Fabry-Perot resonances are also known for noncorrugated slabs of PCs [19,23]. In contrast with solid dielectric slabs, in
PCs we have $v_{g} \neq v_{p h}$ in the general case, where $v_{g}$ and $v_{p h}$ are group and phase velocities, and $n_{g} \neq n_{p h}$, where $n_{g}$ and $n_{p h}$ are group and phase indices of refraction. Location of the minima and maxima of $T$ depend, in fact, on $v_{g}$ and $n_{g}$. An equivalent group index at $\theta=0, n_{g}^{\prime}$, can simply be estimated from the locations of the transmission maxima by using the following formula $[19,22]$ :

$$
n_{g}^{\prime}=\pi(\Delta k D)^{-1},
$$

where $\Delta k$ is the distance between the two neighboring peaks in units of $k$, and $D$ is the thickness of the slab of PC. Generally speaking, assigning a certain value of $D$ to a finite-thickness slab of PC is ambiguous, because it indicates the distance between virtual interfaces. Uncertainty in the introduction of these interfaces in the case of PC cannot be avoided. In addition, it is assumed in Eq. (2) that $n_{g}^{\prime}>0$, which is not always the case. Despite this, the qualitatively correct estimates of $\left|n_{g}\right|$ can also be obtained by using Eq. (2) when $n_{g}^{\prime}<0$. Calculation of the accurate (intrinsic) values of $n_{g}$ for $s$ polarization invokes the modal analysis and postprocessing of its results by using the formulas [31]

$$
\left|n_{g}\right|=c /\left|\mathbf{v}_{\mathbf{g}}\right|
$$

and

$$
\mathbf{v}_{\mathbf{g}}=\left(c^{2} / \omega_{h, \mathbf{k}}\right) \sum_{\mathbf{G}}(\mathbf{k}+\mathbf{G}) E_{\mathbf{G}}^{2}\left(\mathbf{k}, \omega_{h, \mathbf{k}}\right) .
$$

Here, $\mathbf{k}=\mathbf{k}^{\mathbf{P C}}$ is the wave vector of the $h$ th Floquet-Bloch mode of the PC, $\mathbf{G}$ is the reciprocal lattice vector, and $E_{\mathbf{G}}$ is the modal coefficient. The question remains open whether Fabry-Perot resonances can still manifest themselves as the (nearly) total transmission peaks if corrugations are placed at one side or two sides of the noncorrugated slab of PC.

Asymmetric transmission is a fundamental property of structures with broken spatial inversion symmetry. In turn, the use of one-sided corrugations is a natural way to obtain such a breaking. At the same time, breaking the structural symmetry can result in the Fabry-Perot resonances becoming imperfect or disappearing altogether. Thus, the requirement of the strongly pronounced Fabry-Perot resonances can contradict that of the strongly asymmetric transmission in the general case. Forward $\left(T^{\rightarrow}\right)$ and backward $\left(T^{\leftarrow}\right)$ transmittances are given as follows:

$$
T^{\rightarrow}=\sum_{m=-\infty}^{\infty} t_{m}^{\rightarrow}=\sum_{m=-\infty}^{\infty}\left|\tau_{m}^{\rightarrow}\right|^{2} \operatorname{Re}\left(\eta_{m}\right) / \operatorname{Re}\left(\eta_{0}\right)
$$

and

$$
T^{\leftarrow}=\sum_{m=-\infty}^{\infty} t_{m}^{\leftarrow}=\sum_{m=-\infty}^{\infty}\left|\tau_{m}^{\leftarrow}\right|^{2} \operatorname{Re}\left(\eta_{m}\right) / \operatorname{Re}\left(\eta_{0}\right)
$$

where $\eta_{m}=\sqrt{k^{2}-\beta_{m}^{2}}, \beta_{m}=k \sin \theta+2 \pi m / L, k=\omega / c, L$ is the grating period, $t_{m}^{\rightarrow}$ and $t_{m}^{\leftarrow}$ are $m$ th-order forward and backward transmittances (diffraction efficiencies), and $\tau_{m} \overrightarrow{ }$ and $\tau_{m}^{\leftarrow}$ are $m$ th-order forward and backward transmission coefficients.

In accordance with the reciprocity theorem [32], $t_{0} \rightarrow=$ $t_{0}^{\leftarrow}=t_{0}$. However, in the general case $t_{m} \neq t_{m}^{\leftarrow}$ at $|m|>0$ for all of the propagating orders regardless of the material 
that the nonsymmetric grating is made of. For example, in the case when the orders with $|m| \leqslant 1$ are only propagating, the forward transmission $T^{\rightarrow}=t_{0}+t_{-1}+t_{+1}$, and the backward transmission $T \leftarrow=t_{0}^{\leftarrow}+t_{-1}^{\leftarrow}+t_{+1}^{\leftarrow}$. Hence, $T^{\rightarrow} \neq T^{\leftarrow}$ because $t_{-1} \neq t_{-1}^{\leftarrow}$ and $t_{+1} \neq t_{+1}^{\leftarrow}$. An exception may exist, however, for certain values of frequency and $\theta$ at which $T^{\rightarrow}=T^{\leftarrow}$. Note that at $\theta=0, t_{-1}^{\leftarrow}=t_{+1}^{\overleftarrow{ }}$ and $t_{-1}^{\rightarrow}=t_{+1}$. At $\theta \neq 0, t_{-1}^{\leftarrow} \neq t_{+1}^{\leftarrow}$ and $t_{-1} \neq t_{+1}$. Furthermore, one can obtain $t_{+1}^{\leftarrow}=0$ and/or $t_{+1}=0$.

Our goal here is to find operation regimes in which the (nearly) total Fabry-Perot resonance transmission coexists with a high contrast between the forward and backward transmission, while $t_{0}$ may be nonzero. PC gratings are expected to be good candidates for obtaining the desired transmission regime because of the richness of the modal properties and combinations of the values of $n_{g}, n_{p h}$, and impedance $Z$ that are achievable in PCs. It is known that the broadband asymmetric transmission with $t_{m}^{\rightarrow} \neq 0$ and $t_{m}^{\leftarrow}=0$ can be obtained in nonsymmetric PC gratings [4]. Assume that the specific dispersion of a certain Floquet-Bloch mode enables the alternating Fabry-Perot-type minima and maxima in the transmission spectrum. Then, they might appear so that the values of $T^{\leftarrow}$ and $T^{\rightarrow}$ are substantially different at the same resonance in a nonsymmetric PC grating.

Figure 1 presents the general geometry of the problem. The studied PC gratings represent a slab of the two-dimensional square-lattice PC; i.e., it is composed of $S$ layers of circular dielectric rods of diameter $d$ and relative permittivity $\varepsilon_{r}$. The grating period is $L=2 a$, where $a$ is a lattice constant. In fact, it is equal to that of one of the interface layers (labeled by g), which is distinguished from all the other layers (labeled by $\mathrm{r}$ ). The structure is assumed to be infinitely long in the $x$ direction. In the PC gratings like that in Fig. 1, specific dispersion features can lead to the different diffraction scenarios. They include the scenario with zero order being the only order coupled to a Floquet-Bloch wave at the input interface and higher order(s) being propagating in the exit half space bounded by the corrugated interface (scenario A) $[4,5]$, and that with higher orders being the only orders coupled to a Floquet-Bloch wave and only at the corrugated input interface (scenario B) $[4,13,15]$.
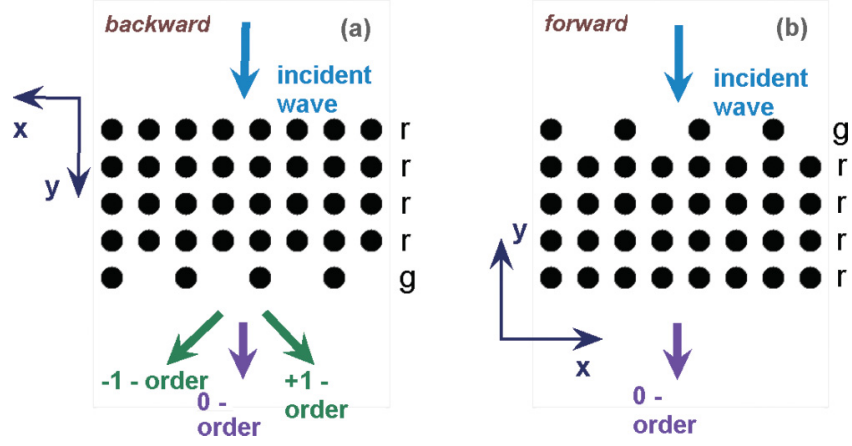

FIG. 1. (Color online) A nonsymmetric PC grating illuminated from (a) the noncorrugated and (b) the corrugated side. Labels $r$ and $g$ denote rod layers corresponding to the regular part and the grating (nonregular) part, respectively; thick arrows schematically show beam paths for scenario A at $\theta=0$.
At $\theta=0$, one can obtain asymmetric transmission with

$$
T^{\rightarrow}=t_{0}
$$

and

$$
T^{\leftarrow}=t_{0}+t_{-1}^{\leftarrow}+t_{+1}^{\leftarrow}
$$

for scenario A, and unidirectional transmission, i.e., asymmetric transmission with

$$
T^{\rightarrow}=\overrightarrow{t_{-1}}+\overrightarrow{t_{+1}}
$$

and

$$
T^{\leftarrow}=0
$$

for scenario $\mathrm{B}$, provided that the orders with $|m| \leqslant 1$ are propagating.

Figure 2 presents an example of transmittance on the $(k L, \theta)$ plane for the PC grating illuminated in a similar way as that in Fig. 1 but at arbitrary $\theta$. The alternating valleys (lower transmittance) and mountains (higher transmittance) are well visible in all of the four plots, indicating Fabry-Perot-type transmission for both $m=0$ and $m=-1$. One can see that scenario A is realizable for both $\theta=0$ and $\theta \neq 0$, while $\theta \neq 0$ is needed here to obtain scenario $\mathrm{B}$. The latter can be realized above the dash-dotted line, while the former can appear below it. The reciprocity manifests itself in that $t_{0}=t_{0}^{\leftarrow}$. On the other hand, $t_{ \pm 1} \neq t_{ \pm 1}^{\leftarrow}$ in the general case. Hence, merging Fabry-Perot resonances with diffractions results in the transmission being asymmetric for scenario A, despite that both $T^{\leftarrow}$ and $T^{\rightarrow}$ are formally allowed to be nonzero for both opposite directions. The mountains of $t_{0}$ and $t_{-1}^{\leftarrow}$ in Figs. 2(a) and 2(b) nearly coincide. This indicates that the -1 order appears at the exit (here corrugated) interface due
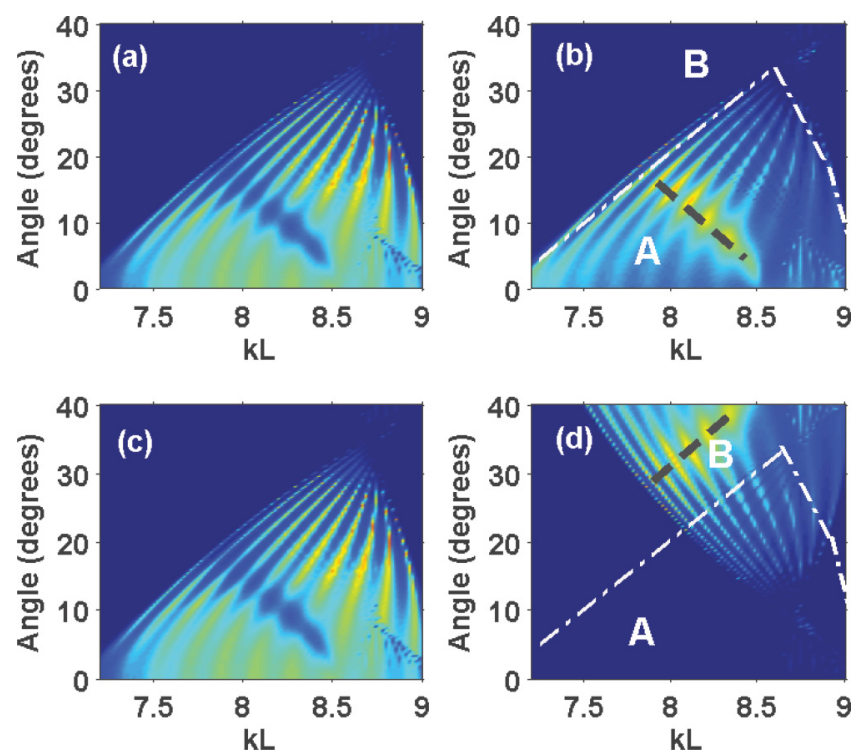

FIG. 2. (Color online) Transmittance on $(k L, \theta)$ plane: (a) $t_{0}^{\leftarrow}$, (b) $t_{-1}^{\leftarrow}$, (c) $t_{0}^{\rightarrow}$, and (d) $t_{-1}^{\rightarrow} ; d / a=0.4, \varepsilon_{r}=11.4, S=12$. One-sided corrugations are obtained by removing every second rod from one of the interface layers of the noncorrugated slab of PC with the same $S$, $L=2 a$. Black dashed lines connect the maxima of $t_{-1}^{\leftarrow}$ (b) and $t_{-1}$ (d) for different mountains; white dash-dotted lines show the approximate boundary below which $t_{0} \neq 0$, the fourth-lowest Floquet-Bloch mode. 
to diffractions while being uncoupled at the input interface. Furthermore, for this scenario, $T^{\leftarrow}$ can be large mainly due to the contribution of $t_{-1}^{\leftarrow}$. For scenario B, the mountains of $t_{0}$ and $t_{-1}^{\rightarrow}$ show different slopes. This corresponds to the different signs of refraction for the orders with $m=0$ and $m=-1$, which are both coupled to the Floquet-Bloch wave at the input (here corrugated) interface. In this paper, we focus on the special case of scenario $A$ that is realized at $\theta=0$, i.e., when $t_{-n}^{\rightarrow}=t_{+n}^{\rightarrow}$ and $t_{-n}^{\leftarrow}=t_{+n}^{\leftarrow}$.

\section{RESULTS AND DISCUSSION}

\section{A. Basic transmission effects}

Let us consider the basic effects associated with asymmetric transmission that may appear while zero order is coupled to a Floquet-Bloch wave. Figure 3 presents transmittance vs $k L$ through the same nonsymmetric PC grating as in Fig. 2, but only at $\theta=0$. For the comparison, transmittance is also shown for the corresponding noncorrugated slabs of PC. In Fig. 3(a), the multiple Fabry-Perot-type maxima of $T^{\leftarrow}=1$ are observed. Such maxima are typical for the noncorrugated dielectric slabs, as well as for some transmission regimes in the noncorrugated slabs of PC. In the considered case, the maxima of $T^{\leftarrow}=1$ appear while one of the interfaces is corrugated so that higher order(s) may contribute to the transmission. It is seen that the orders with $m= \pm 1$ together take more than $50 \%$ of the incident-wave energy, i.e., $T^{\leftarrow /} T^{\rightarrow}>2$. Contribution
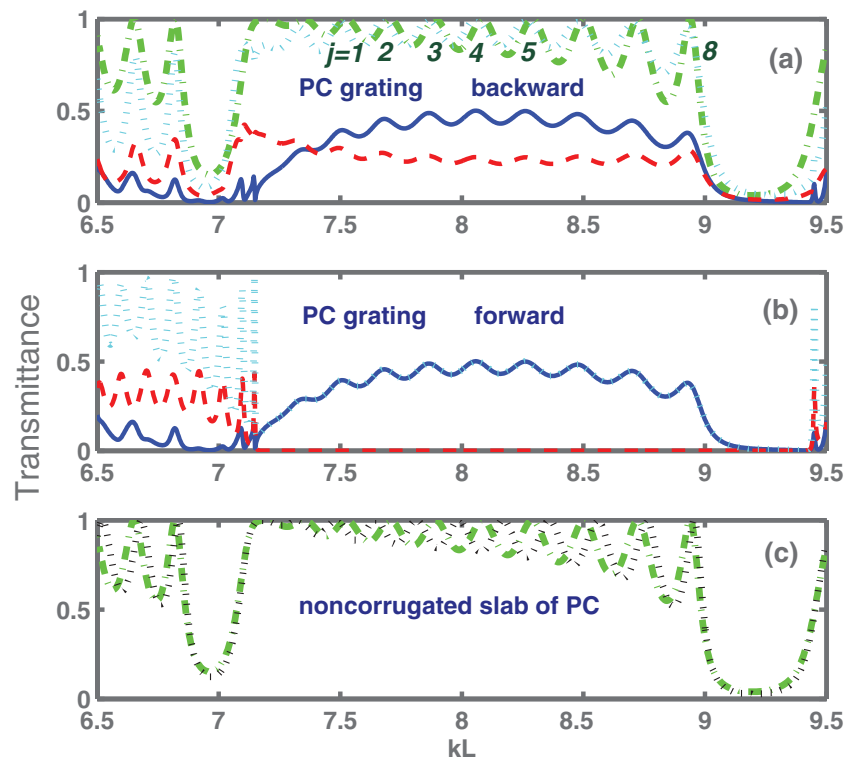

FIG. 3. (Color online) Transmittance vs $k L$ at $d / a=0.4, \varepsilon_{r}=$ 11.4, $S=12$, and $\theta=0$ for the fourth-lowest Floquet-Bloch mode $(7.1<k L<9.04)$ and partially for the neighboring modes of the PC grating at (a) noncorrugated-side $\left(T^{\leftarrow}\right)$ and (b) corrugated-side $\left(T^{\rightarrow}\right)$ illumination, and (c) for the noncorrugated slab of PC $\left(T^{\rightarrow}=T^{\leftarrow}=\right.$ $t_{0}$ ). Corrugations are obtained by removing every second rod from the rod layer that is adjacent to one of the interfaces, $L=2 a(\mathrm{a})$, (b); blue solid line, $t_{0}$ (a), (b); red dashed line, (a) $t_{-1}^{\leftarrow}=t_{+1}^{\leftarrow}$ and (b) $t_{-1}=t_{+1}$; (a), (b) cyan dotted line, sum over all of the propagating orders; (a), (c) green dash-dotted line and (c) black dotted line, transmittance for the noncorrugated slab of PC at $S=11$ and $S=12$, respectively. The maxima are numbered starting from $k L=7.51(j=1)$. of higher orders to the transmission strongly depends on the incidence side, while $t_{0}^{\leftarrow}=t_{0}^{\rightarrow}$. Reflection-free splitting can appear only at the noncorrugated-side illumination, when higher orders contribute to the transmission due to the effect of the corrugated exit interface.

As expected, locations of the maxima of $T \leftarrow$ in Fig. 3(a) and $T \rightarrow$ in Fig. 3(b) differ just slightly. From the comparison of Figs. 3(a) and 3(b), three important features can be seen: (i) only zero order, which is associated with symmetric transmission, is expected to be coupled to the Floquet-Bloch wave at the input interface, regardless of the illumination side; (ii) umklapp scattering does not provide coupling of higher diffraction orders to a Floquet-Bloch wave at the corrugated input interface and, thus, does not contribute to $T^{\rightarrow}$; and (iii) higher orders may propagate in the exit half-space due to the corrugated exit interface and contribute to $T^{\leftarrow}$ while $T^{\rightarrow}=t_{0}$.

Figure 3(c) presents transmittance for the noncorrugated slabs of PC. Locations of the maxima of $T^{\leftarrow}$ and $T^{\rightarrow}$ for the PC grating in Figs. 3(a) and 3(b) nearly coincide with those of $T=t_{0}$ at $S=11$ (the thinner noncorrugated slab of PC) in Fig. 3(c), rather than with those of $T=t_{0}$ at $S=12$ (the thicker noncorrugated slab of PC). In fact, the corrugations "reduce" the effective thickness of the PC grating in the totaltransmission regime. Thus, the main role of the corrugated exit layer here is to enable the propagation regime for some higher orders, which are themselves not coupled to the Floquet-Bloch wave at the exit interface. Their significant contribution to the transmission originates rather from the specific coupling of the diffraction orders in the exit half space to each other. In turn, the regular part of the PC grating is responsible for the appearance of Fabry-Perot-type resonances that are associated with the maxima of $T^{\leftarrow}=1$. Surprisingly, contributions of these two parts of the entire structure to the transmission can show (nonideal) additivity. Furthermore, this is a one-way effect; i.e., it does not remain for the opposite incidence direction.

Then, $n_{g}^{\prime}$ has been estimated using Eq. (2) for the $k L$ range corresponding to the fourth-lowest Floquet-Bloch mode. In particular, $n_{g}^{\prime}$ in Fig. 3(a) varies from 2.99 to 2.7 between the peaks labeled by $j=1$ and $j=4$, and from 2.55 to 2.31 between the peaks labeled by $j=4$ and $j=8$. It is noteworthy that scenario $\mathrm{A}$ at $\theta=0$ can be obtained also in the earlier studied metallic slabs with the branched waveguides that connect the input and output interfaces [33], one-dimensional PCs with one-side corrugations [16], and nonsymmetric gratings made of homogeneous Drude materials [34]. In contrast to the PC gratings, it is not clear yet whether the maxima of $T^{\leftarrow}=1$ can be obtained in these structures.

Figure 4 presents the equifrequency contours (EFCs) in $\mathbf{k}$ space at $k L=8.1$ and construction lines for the parameters of the PC grating in Fig. 3. In order to couple a desired order to the Floquet-Bloch wave of the $\mathrm{PC}$, conservation of the tangential wave vector component, $k_{x, m}$, is required, which is possible if the EFC of the PC crosses the construction line plotted at the same $k L$ value [4,31]. Locations of the construction lines are given by

$$
k_{x, m}=k \sin \theta+2 \pi m / L .
$$

It is seen that zero order is coupled to the Floquet-Bloch wave in a two-way manner. The orders with $m= \pm 1$ may propagate 


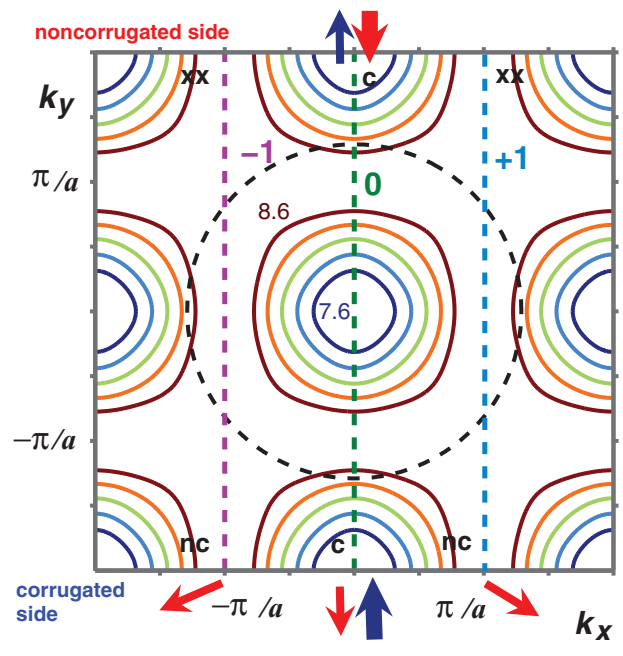

FIG. 4. (Color online) EFCs of the infinite PC with $d / a=0.4$ and $\varepsilon_{r}=11.4$ for $k L=7.6,7.85,8.1,8.35$, and 8.6. Dashed circle, EFC in air at $k L=8.1$; vertical dashed lines, construction lines; " -1, , " 0 ," and " +1 " indicate the order $(m)$; "c" and " $x x$ " at plot top denote that the corresponding order is either coupled to the Floquet-Bloch mode at the noncorrugated input interface, or evanescent in the adjacent air half space, respectively; "c" and "nc" at plot bottom denote that the corresponding order either is coupled to the Floquet-Bloch mode at the corrugated input interface, or may propagate in the adjacent air half space but is not coupled itself to the Floquet-Bloch mode. Thick and thin arrows schematically show directions of the incident and transmitted beams, respectively; for convenience, orientation of the axes of $k_{x}$ and $k_{y}$ corresponds to the directions of the $x$ and $y$ axes which are opposite those in Fig. 1.

in the exit half space at the noncorrugated-side illumination owing to the corrugations placed at the exit interface. However, they remain evanescent in the half space that is adjacent to the noncorrugated exit interface at the corrugated-side illumination. The presented EFC results coincide well with the transmission results in Fig. 3. Asymmetric coupling and asymmetric diffraction can be understood from the following. If the exit interface has a larger period than the input one (e.g., $L=2 a, 3 a, 4 a$, etc.), new diffraction orders can appear in transmission as compared to those coupled to the Floquet-Bloch wave at the input interface, for which $L=a$. However, if the input interface has a larger period, transmission channels that are open due to coupling at the input interface cannot be closed at the exit interface having the smaller period. The new orders may include those coupled to the Floquet-Bloch wave at the exit interface both directly and not directly, i.e., as at the noncorrugated-side illumination in Fig. 4.

At the edges of the $k L$ range corresponding to the fourthlowest Floquet-Bloch mode, the coupling scenarios are not restricted to that demonstrated in Fig. 4. For example, at the lower- $k L$ edge, the third and fourth modes can contribute to $T \leftarrow$ simultaneously. The shape, size, and location of the EFCs allow to introduce the approximate equivalent index of refraction, $0.24<n_{p h}<0.52$, at least at $7.6<k L<8.35$, where $n_{p h}$ can be several times smaller than $n_{g}$. Hence, although the values of $n_{p h}$ belong here to the range that is associated with Drude metals above the plasma frequency and wire media above the effective plasma frequency, in the general
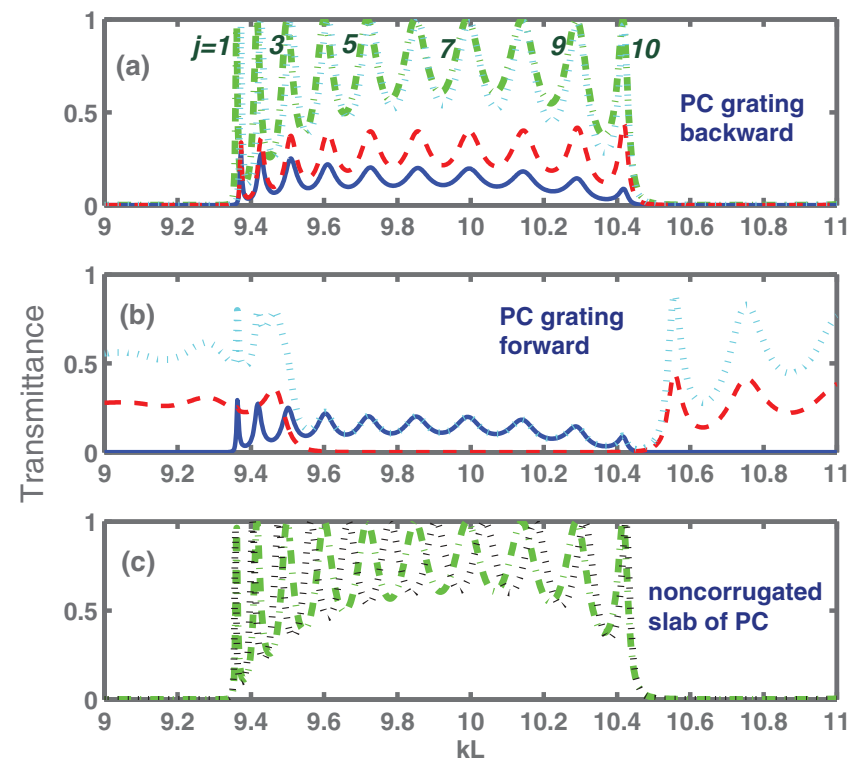

FIG. 5. (Color online) Same as Fig. 3 but for the third-lowest Floquet-Bloch mode and partially for the neighboring modes at $\varepsilon_{r}=5.8$.

case it is impossible to reproduce the observed dependencies of $T^{\rightarrow}$ and $T^{\leftarrow}$ for a slab that is made of a dispersionless homogeneous material with the same $n_{p h}$ and has similar corrugations, because in such a case $n_{p h}=n_{g}$.

Adjusting the PC lattice and corrugation parameters, we can obtain the case when the contribution of higher orders to $T^{\leftarrow}$ is substantially stronger than that in Fig. 3 and, hence, the contrast $T^{\leftarrow /} T^{\rightarrow}$ is much higher, while $T^{\rightarrow}=t_{0}$. Figure 5 presents an example of the Fabry-Perot-type transmission with the multiple transmittance maxima for the third-lowest Floquet-Bloch mode. Now, the orders with $m= \pm 1$ provide the main contribution to $T^{\leftarrow}$ and $T^{\leftarrow /} T^{\rightarrow}>10$ at the upper edge of the band. This also occurs at the near-edge peak at $k L=10.418$, which is labeled by $j=10$.

Comparing the peak locations in Fig. 5 for the corrugated slab with $S=12$ and the noncorrugated slab with $S=11$, one can see that the additivity mentioned in Fig. 3 remains, whereas the difference now tends to vanish. In fact, the effect exerted by the nonregular interface layer on the Fabry-Perot resonances arising within the regular part of the PC grating disappears. At the lower edge of the band, $t_{0} \approx t_{+1}^{\leftarrow}=t_{-1}^{\leftarrow}$ within a wide $k L$ range, including the peak of $T^{\leftarrow}$ labeled by $j=1$. Hence, splitting can appear while reflections are vanishing. However, the second- and third-lowest passbands are overlapped in the vicinity of $k L=9.5$; compare Figs. 5(a) and 5(b). Thus, this splitting regime is not one way. Table I presents the values of $n_{g}^{\prime}$ obtained using Eq. (2). $k_{l} L$ and $k_{s} L$ denote the larger and smaller values of $k L$, respectively, for every pair of the neighboring peaks of $T^{\leftarrow}$. The smaller the distance between the neighboring peaks, the larger $n_{g}^{\prime}$ is.

Figure 6 presents electric field distribution at the peak of $T^{\leftarrow}$ labeled by $j=10$ in Fig. 5(a). It is seen that the field distribution in the regular part of the PC grating at the noncorrugated-side illumination and that in the noncorrugated slab of the PC with the same number of rod layers as the regular part of the PC grating $(S=11)$ are almost identical 
TABLE I. Equivalent index of refraction $n_{g}^{\prime}$ estimated from locations of the peaks of $T^{\leftarrow}$ in Fig. 5(a).

\begin{tabular}{lccc}
\hline \hline$j, j+1$ & $k_{s} L$ & $k_{l} L$ & $n_{g}^{\prime}$ \\
\hline 1,2 & 9.379 & 9.434 & 9.52 \\
2,3 & 9.434 & 9.514 & 6.54 \\
3,4 & 9.514 & 9.615 & 5.18 \\
4,5 & 9.615 & 9.73 & 4.55 \\
5,6 & 9.73 & 9.86 & 4.03 \\
6,7 & 9.86 & 10 & 3.74 \\
7,8 & 10 & 10.147 & 3.56 \\
8,9 & 10.147 & 10.295 & 3.54 \\
9,10 & 10.295 & 10.418 & 4.2 \\
\hline \hline
\end{tabular}

[compare Figs. 6(a) and 6(d)]. These results are in agreement with the above discussed additivity. Strong field confinement takes place inside certain regions (between the rods), where its magnitude is 9.5 times larger than that of the incident wave. The same remains true for the noncorrugated slab of the PC with $S=12$ in Fig. 6(c), which is out of resonance at the used value of $k L$. In the case shown in Fig. 6(b), there are strong reflections at the corrugated input interface.

It is noteworthy that the unidirectional transmission with $T^{\rightarrow}=t_{-1}+t_{+1}$ and $T^{\leftarrow}=0$ appears in the adjacent $k L$ ranges, i.e., at $k L<9.34$ and $k L>10.45$ [see Fig. 5(b)]. At $k L=10.45$, switching between the two strongly asymmetric, diodelike operation regimes with $t_{-1}^{\leftarrow}+t_{+1}^{\leftarrow}>0.9$ and $t_{-1}+$ $t_{+1}>0.9$ can be realized at a weak variation in $k L$, i.e., from $k L=10.418$ to $k L=10.56$ (1.42\% difference in value of (a)

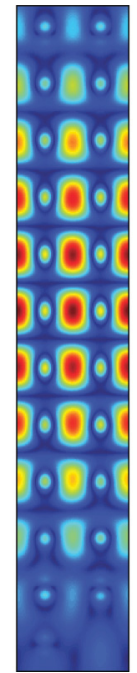

(b)

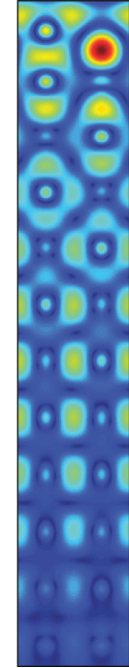

(c)

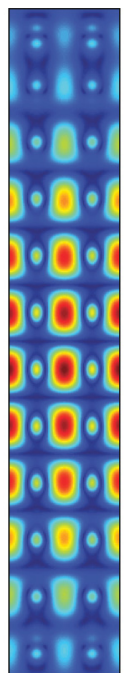

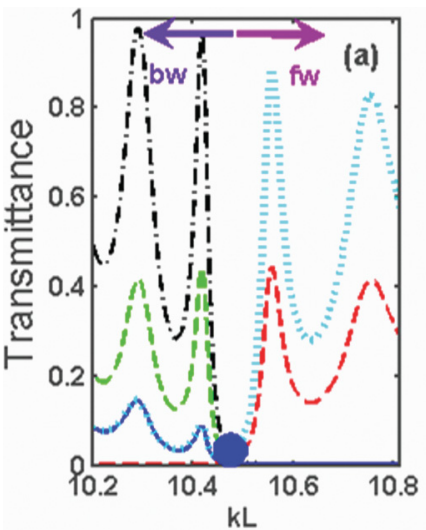

(c)

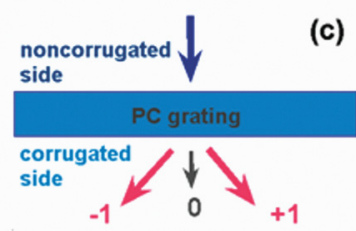

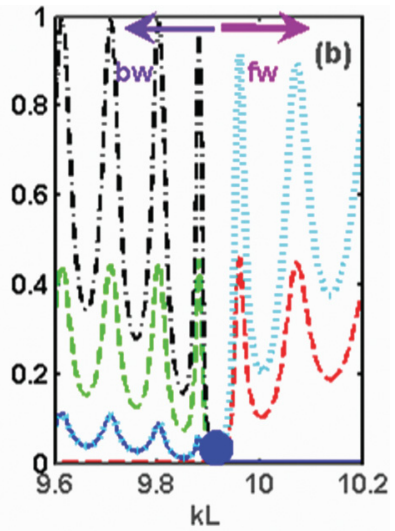

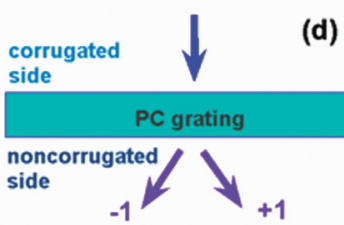

FIG. 7. (Color online) Transmittance vs $k L$ at switching between two asymmetric regimes for (a) the same PC grating as in Figs. 5(a) and 5(b), and (b) at $d / a=0.32, \varepsilon_{r}=9.61, S=16$, and corrugations obtained by removing every second rod from one of the interface layers of the noncorrugated slab of PC; $\theta=0$, blue solid line, $t_{0}=$ $t_{0}^{\leftarrow}=t_{0}^{\rightarrow}$; green dashed line, $t_{ \pm 1}^{\leftarrow}$; red dashed line, $t_{ \pm 1}^{\rightarrow}$; black dashdotted line, $T^{\leftarrow}$; cyan dotted line, $T^{\rightarrow}$; circles, vicinity of $k L$ value, at which the switching takes place; (c), (d) schematics of the incident and transmitted beam paths for backward (bw) and forward (fw) transmission, respectively.

$k L)$. The corresponding diffraction angles are $\phi_{ \pm 1}= \pm 37^{\circ}$ and $\phi_{ \pm 1}= \pm 36.5^{\circ}$. A fragment of the $k L$ dependence of transmittance from Figs. 5(a) and 5(b) is shown in Fig. 7(a) to demonstrate the details of this regime. The possibility of a further decrease of the distance between the near-edge peaks of $T^{\rightarrow}$ and $T^{\leftarrow}$ is demonstrated in Fig. 7(b). Here, the peaks are located at $k L=9.96\left(T^{\rightarrow}\right)$ and $k L=9.88\left(T^{\leftarrow}\right)$, so that there is only a $0.8 \%$ difference. Note that asymmetry in the Fabry-Perot-type transmission, which corresponds to scenario B, is observed in Figs. 7(a) and 7(b) rightward from the switching points.

Figure 8 presents the EFCs and construction lines for the PC grating from Fig. 5. All the contours shown belong to the third-lowest Floquet-Bloch mode. The coupling scenario is distinguished from that in Fig. 4. Now, the orders with $m=0, \pm 1$ are allowed to couple to the Floquet-Bloch mode at the corrugated input interface. However, as follows from the comparison with Fig. 5(b), transmission channels associated with these orders do not contribute to the transmission. This regime will be studied in detail in a future paper. At the noncorrugated-side illumination, the order with $m=0$ is the only order that may be coupled to the Floquet-Bloch wave at the input interface. It is noteworthy that $\mathbf{S} \cdot \mathbf{k}^{\mathbf{P C}}>0$ for (near-)square EFCs centered around the $\Gamma$ point $\left(k_{x}=k_{y}=\right.$ 0 ), where coupling of zero order is possible, but $\mathbf{S} \cdot \mathbf{k}^{\mathbf{P C}}<0$ for the (near-)circular EFCs around the $M$ point, where coupling to the $m= \pm 1$ order might be possible at the corrugated-side illumination. The comparison of Figs. 5 and 8 gives an example of how the well-pronounced asymmetry in the Fabry-Perot-type 


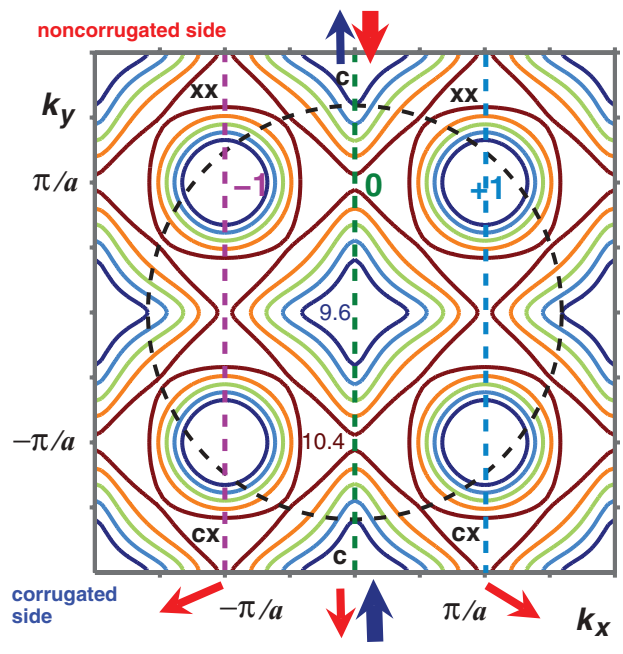

FIG. 8. (Color online) Same as Fig. 4 but for the infinite PC with $d / a=0.4$ and $\varepsilon_{r}=5.8$, at $k L=9.6,9.8,10,10.2$, and 10.4. EFC in air is shown for $k L=10$; "cx" stands for the case when coupling of a certain order is formally allowed but it does not contribute to transmission.

transmission can be obtained for various EFC shapes. The presented results show that the EFC-based analysis can be insufficient to predict the correct scenario of asymmetric transmission.

\section{B. Effect of corrugation depth and thickness}

Now let us demonstrate the basic effects of connection with variations in corrugation depth and thickness of the $\mathrm{PC}$ grating. Figure 9 illustrates the effect exerted by the depth of one-sided corrugations on $t_{0}$ and $t_{ \pm 1}^{\leftarrow}$, for the same PC lattice parameters and the same Floquet-Bloch mode as in Figs. 5 and 8. First, we increase the corrugation depth by removing every second rod from one more layer and the same columns as in Figs. 5(a) and 5(b). In other words, we take a thinner regular part and a thicker grating part, while the total thickness is kept $(S=12)$. This results in strong variation in $t_{0}$ and $t_{ \pm 1}^{\leftarrow}$ at the peaks of $T^{\leftarrow}$. In contrast to Fig. 5(a), symmetric transmission $\left(t_{0}\right)$ dominates now in $T^{\leftarrow}$ at the upper edge of the transmission band [see Fig. 9(a)]. A further increase of the corrugation depth leads to the suppression of the first-order transmittance at $9.35<k L<9.9$, where it may be nonzero, as seen in Fig. 9(b). Finally, Fig. 9(c) shows that at the same thickness of the regular and grating parts transmission is suppressed at the peak at $k L=9.975$; i.e., $t_{0}^{\leftarrow}=$ $t_{0}^{\rightarrow}=0$ and $T^{\leftarrow}=t_{-1}^{\leftarrow}+t_{+1}^{\leftarrow}$, while $T^{\rightarrow}=0$. Here, extremely strong asymmetry is achieved. Since transmission is zero in one direction, it is similar to scenario B in Fig. 2, but it is distinguished from it in that now $T^{\leftarrow}=1$ and $T^{\rightarrow}=0$. On the other hand, it is similar to scenario A in Fig. 2, but now $T^{\leftarrow}=1$. This regime is associated with the anomalous conversion of the incident wave energy into that of the transmitted higher orders.

Comparison of Figs. 9(a), 9(b), and 9(c) illustrates the idea of reconfigurable structures, in which removing or adding some rods can result in complete redistribution of the transmitted energy in favor of either higher orders or zero order, while the resonance frequency is just slightly shifted. For example, $T^{\leftarrow}=t_{0}=1$ at $k L=9.453$ in Fig. 9(b), while
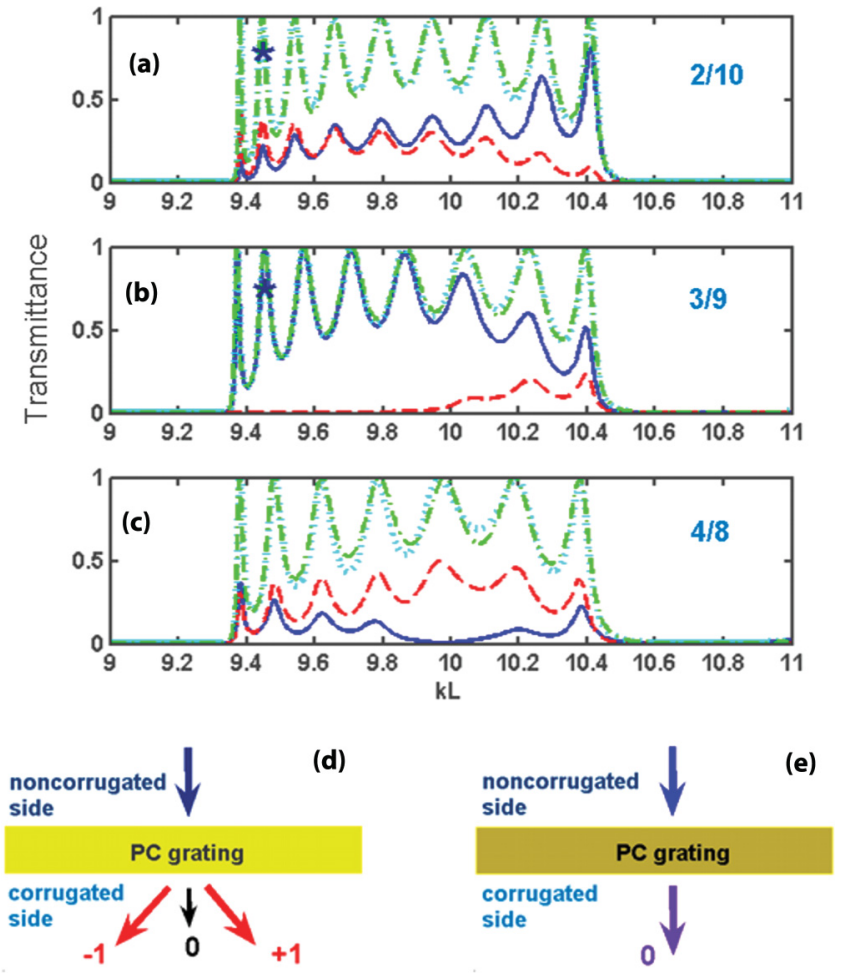

FIG. 9. (Color online) Same as Fig. 5(a), except for deeper corrugations: rods are removed from (a) two, (b) three, and (c) four layers adjacent to the exit interface. Results for the noncorrugated slab of PC are shown by a green dash-dotted line for (a) $S=10$, (b) $S=9$, and (c) $S=8$. Pairs of numbers in each plot show the number of rod layers in the grating (nonregular) and regular parts of the PC grating; (d) and (e) schematically show the incident and transmitted beam paths in cases labeled by an asterisk in (a) and (b), respectively.

$T^{\leftarrow}=t_{0}+t_{-1}+t_{+1}=1$ at $k L=9.446$ in Fig. 9(a) (these cases are denoted by *). Note that the number of the peaks of $T^{\leftarrow}$ varies in Fig. 9 depending on the thickness of the regular part of the PC. Similarly to Figs. 3 and 5, their locations for the PC grating nearly coincide with those for the noncorrugated slabs of PC with the same number of the rod layers as the regular part of the PC grating. Hence, the additivity can occur also at deep corrugations.

Figure 10 presents transmittance as a function of $k L$ for PC gratings that have the same parameters as in Fig. 5(a), except for the values of $S$. The general trend in behavior of transmittance with varying structure thickness is quite predictable: the number of the total-transmission maxima increases with $S$. Again, the only principal difference between $T^{\leftarrow}$ for the PC grating and $T^{\leftarrow}=T^{\rightarrow}$ for the noncorrugated slab of PC with the same $S$ as the regular part of the PC grating is the contribution of higher orders in the former case.

It is interesting that the variation in $S$ just slightly affects the contribution of the individual orders. Since all the peaks become narrower and the near-edge peaks are located closer to the band edges as $S$ is increased, stronger contribution of $t_{ \pm 1}^{\leftarrow}$ to $T^{\leftarrow}$ might be possible. This feature is observed in Fig. 10(d). Here, $T^{\leftarrow} \approx 1$ and $T^{\leftarrow / T^{\rightarrow}}=15.1$ at $k L \approx 10.46$. In turn, 

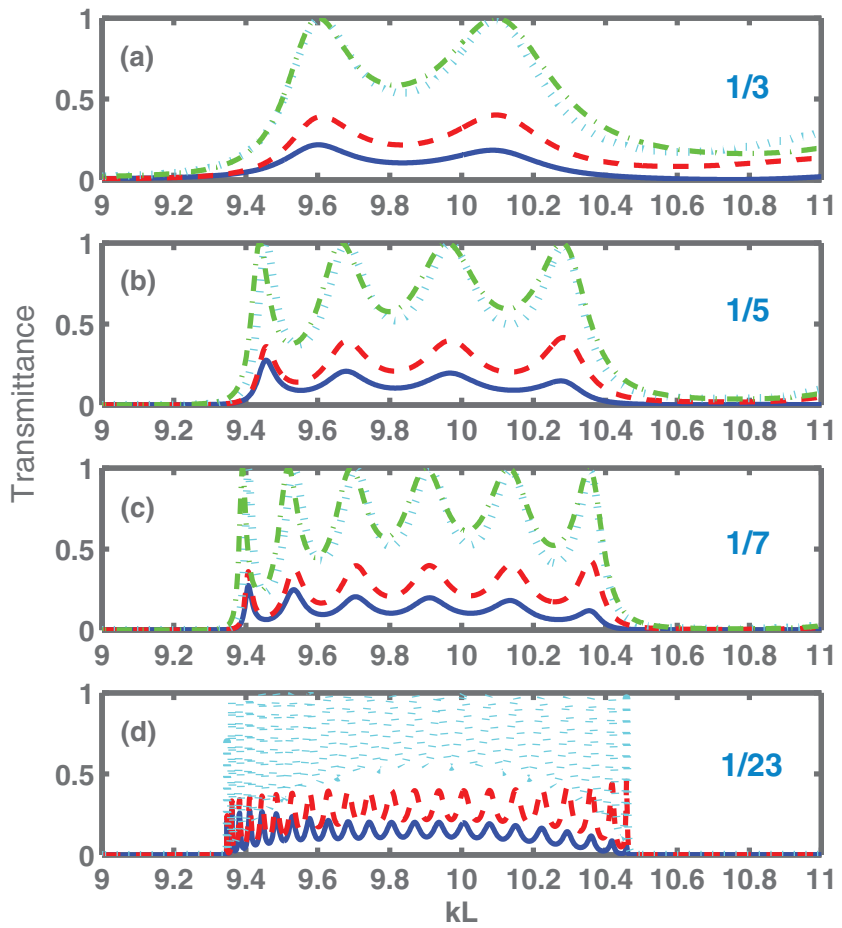

FIG. 10. (Color online) Same as Fig. 5(a) but for (a) $S=4$, (b) $S=6$, (c) $S=8$, and (d) $S=24$. Results for the noncorrugated slab of the PC are shown by green dash-dotted line for (a) $S=3$, (b) $S=5$, and (c) $S=7$. Pairs of the numbers in each plot show the number of rod layers in the grating and regular parts of the PC grating.

$n_{g}^{\prime}$ is increased while the band edge is approached. Thus, the larger $n_{g}^{\prime}$, the larger $T^{\leftarrow /} T^{\rightarrow}$ is. Equation (2) gives $n_{g}^{\prime} \approx 6.4$ for the two neighboring peaks at the right edge of the band. Accordingly, the near-edge peaks can show a larger value of $Q$ factor. For example, in Fig. $10(\mathrm{~d}), Q=2.1 \times 10^{3}$ for the peak at $k L \approx 10.46$.

\section{One-way splitting}

Two applications of the studied mechanism of asymmetrical transmission-switchable diodelike transmission and PC gratings that are reconfigurable due to the varying corrugation depth-have already been discussed. In the previous studies of PC gratings, one-way splitting has been demonstrated for the two outgoing beams $(m= \pm 1)$, while transmission at the noncorrugated-side illumination is blocked [4]. A similar but inverse regime, i.e., that with $T \leftarrow=t_{-1}^{\leftarrow}+t_{+1}^{\leftarrow} \neq 0$ and $T^{\rightarrow}=0$, has been observed in Fig. 9(c) at $k L=9.975$. Figure 11 demonstrates reflection-free one-way splitting in the inverse regime with three outgoing beams, i.e.,

$$
T^{\leftarrow}=t_{0}+t_{-1}^{\leftarrow}+t_{+1}^{\leftarrow}=1
$$

In this case, $T^{\rightarrow}=t_{0} ; t_{0} \approx t_{-1}^{\leftarrow}=t_{+1}^{\leftarrow}$ can remain in a wide $k L$ range where $T \leftarrow \neq 1$, as in Fig. 11(a). This feature is important in case of nonmonochromatic incident waves, despite that total transmission cannot be obtained. On the other hand, PC grating in Fig. 11(c) allows one to keep $T^{\leftarrow}>0.85$ in the vicinity of the regime labeled by the arrow. Hence, even if splitting
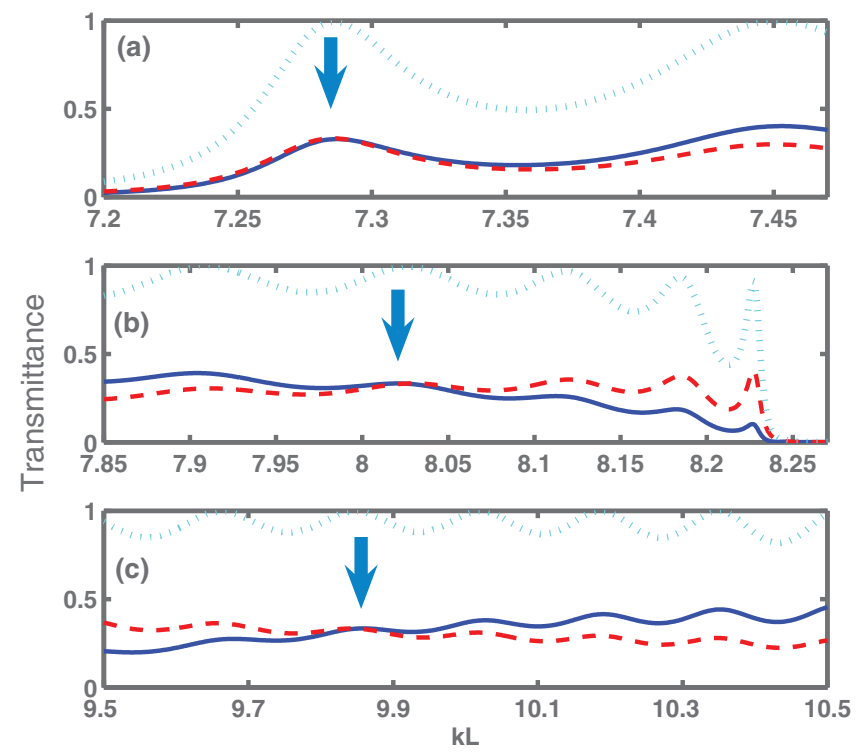

FIG. 11. (Color online) Transmittance vs $k L$ for three PC gratings with $\varepsilon_{r}=9.61$ and $S=12$, in which splitting with $t_{0}=t_{-1}^{\leftarrow}=t_{+1}^{\leftarrow}$ (arrows) is realized at $\theta=0$; (a) $d / a=0.45$, (b) $d / a=0.55$, and (c) $d / a=0.4$ and $d_{1} / a=0.3$ for the outer and inner radii of annular rods, respectively. Corrugations are obtained by removing every second rod from one of the interface layers of the noncorrugated slab of PC; blue solid line, $t_{0}$; red dashed line, $t_{ \pm 1}^{\leftarrow}$; and cyan dotted line, $T^{\leftarrow}$.

is imperfect, i.e., $t_{0} \neq t \overleftarrow{ \pm 1}$, reflections at nonmonochromatic illumination are expected to be rather low.

\section{CONCLUSIONS}

The potential of nonsymmetric PC gratings based on two-dimensional dielectric PCs in the strongly asymmetric transmission has systematically been studied for the scenarios when zero order, which is associated with symmetric transmission, is coupled to the Floquet-Bloch wave at normal incidence. This mechanism needs asymmetry in coupling and, hence, nonequal contributions of higher diffraction orders to the forward transmission (corrugated-side illumination) and the backward transmission (noncorrugated-side illumination). Since zero-order transmission is symmetric, the forward and the backward transmission can be strongly distinguished when higher orders provide the dominant contribution to the latter. Fabry-Perot resonances associated with the total-transmission maxima observed in PC gratings in the backward case are inherited from those of the noncorrugated slab of PC with the same number of rod layers as the regular part of the PC grating, while the grating (nonregular) part, which is placed at the exit side, generally plays the role of a splitter. Furthermore, unidirectionality with the vanishing forward transmission and total backward transmission can be obtained. Asymmetric Fabry-Perot-type transmission can coexist with large values of the equivalent group index of refraction so that the waves being quite slow inside a PC can be strongly coupled to higher diffraction orders, which are the main contributors to the backward transmission. One-way splitting with the desired nonequal or equal contributions of two or three 
transmitted beams, and switching of the direction of strong transmission in the asymmetric regime, are two examples of possible applications of the studied mechanism. Varying the corrugation depth can be an efficient tool for controlling the contributions of the individual diffraction orders also when asymmetric transmission is free of reflections for one of the two opposite incidence directions. The obtained results can be used in a wide frequency range which extends from acoustic to optical frequencies.

\section{ACKNOWLEDGMENTS}

This work is supported by the projects DPT-HAMIT, ESFEPIGRAT, NATO-SET-181, and TUBITAK under Projects No. 107A004, No. 109A015, and No. 109E301. A.E.S. thanks Deutsche Forschungsgemeinschaft for partial support of this work under Project No. SE1409/2-2. One of the authors (E.O.) also acknowledges partial support from the Turkish Academy of Sciences.
[1] D. Maystre, Opt. Express 8, 209 (2001).

[2] S. Collardey, A.-C. Tarot, P. Pouliguen, and K. Mahdjoubi, Microwave Opt. Technol. Lett. 44, 546 (2005).

[3] A. E. Serebryannikov, T. Magath, and K. Schuenemann, Phys. Rev. E 74, 066607 (2006).

[4] A. E. Serebryannikov, Phys. Rev. B 80, 155117 (2009).

[5] A. Mandatori, M. Bertolotti, and C. Sibilla, J. Opt. Soc. Am. B 24, 685 (2007).

[6] F. D. M. Haldane and S. Raghu, Phys. Rev. Lett. 100, 013904 (2008).

[7] Z. Yu, Z. Wang, and S. Fan, Appl. Phys. Lett. 90, 121133 (2007).

[8] M. Scalora, J. P. Dowling, C. M. Bowden, and M. J. Bloemer, J. Appl. Phys. 76, 2023 (1994).

[9] A. B. Khanikaev, S. H. Mousavi, G. Shvets, and Y. S. Kivshar, Phys. Rev. Lett. 105, 126804 (2010).

[10] A. Figotin and I. Vitebskiy, Phys. Rev. B 67, 165210 (2003).

[11] K. B. Alici, F. Bilotti, L. Vegni, and E. Ozbay, Opt. Express 17, 5933 (2009).

[12] K. B. Alici, A. E. Serebryannikov, and E. Ozbay, J. Electromag. Waves Appl. 24, 1183 (2010).

[13] A. E. Serebryannikov, A. O. Cakmak, and E. Ozbay, Opt. Express 20, 14980 (2012).

[14] S. Xu, C. Qiu, and Z. Liu, J. Appl. Phys. 111, 094505 (2012).

[15] A. O. Cakmak, E. Colak, A. E. Serebryannikov, and E. Ozbay, Opt. Express 18, 22283 (2010).

[16] X.-B. Kang, W. Tan, Z.-S. Wang, Z.-G. Wang, and H. Chen, Chin. Phys. Lett. 27, 074204 (2010).

[17] C. Lu, X. Hu, H. Yang, and Q. Gong, Opt. Lett. 36, 4668 (2011).

[18] E. Plum, V. A. Fedotov, and N. I. Zheludev, Appl. Phys. Lett. 94, 131901 (2009); R. Singh, E. Plum, C. Menzel, C. Rockstuhl, A. K. Azad, R. A. Cheville, F. Lederer, W. Zhang, and N. I. Zheludev, Phys. Rev. B 80, 153104 (2009); M. Mutlu, A. E. Akosman, A. E. Serebryannikov, and E. Ozbay, Phys. Rev. Lett. 108, 213905 (2012).
[19] K. Sakoda, Optical Properties of Photonic Crystal (Springer, Berlin, 2001).

[20] J. H. Wu, L. K. Ang, A. Q. Liu, H. G. Teo, and C. Lu, J. Opt. Soc. Am. B 22, 1770 (2005); X. Chen, D. Zhao, Z. Qiang, G. Lin, H. Li, Y. Qiu, and W. Zhou, Appl. Opt. 49, 5878 (2010); E. N. Bulgakov and A. F. Sadreev, Phys. Rev. B 81, 115128 (2010).

[21] J. Cos, J. Ferre-Borrull, J. Palares, and L. F. Marsal, Opt. Commun. 282, 1220 (2009); R. Iliew, C. Etrich, T. Pertsch, F. Lederer, and K. Staliunas, Opt. Lett. 33, 2695 (2008); A. Saeynaetjoki, M. Mulot, and S. Arpiainen, J. Opt. A 8, S502 (2006).

[22] T. Baba, Nat. Photonics 2, 465 (2008).

[23] A. E. Serebryannikov, E. Ozbay, and P. V. Usik, Phys. Rev. B 82, 165131 (2010).

[24] A. Figotin and I. Vitebskiy, Phys. Rev. A 76, 053839 (2007); M. Mulot, A. Saeynaetjoki, S. Arpiainen, H. Lipsanen, and J. Ahopelto, J. Opt. A 9, 5415 (2007).

[25] K. Kiyota, T. Kisa, N. Yokouchi, T. Ide, and T. Baba, Appl. Phys. Lett. 88, 201904 (2006).

[26] A. E. Serebryannikov and E. Ozbay, Opt. Express 17, 13335 (2009).

[27] This is possible because zero order is insensitive to breaking spatial inversion symmetry.

[28] T. Magath and A. E. Serebryannikov, J. Opt. Soc. Am. A 22, 2405 (2005).

[29] See www.cst.com

[30] M. Born and E. Wolf, Principles of Optics, 6th ed. (Pergamon, Oxford, 1980), Sec. 7.6.1.

[31] S. Foteinopoulou and C. M. Soukoulis, Phys. Rev. B 72, 165112 (2005).

[32] J. A. Kong, Electromagnetic Wave Theory (EMW Publishing, Cambridge, MA, 2005).

[33] M. J. Lockyear, A. P. Hibbins, K. R. White, and J. R. Sambles, Phys. Rev. E 74, 056611 (2006).

[34] A. E. Serebryannikov and E. Ozbay, Opt. Express 17, 278 (2009). 